\title{
Design of Camouflage Pattern Based on Mathematical Morphology
}

\author{
Qi Jia, Xitong Yang*, Weidong Xu, Xuliang Lv and Jianghua $\mathrm{Hu}$ \\ College of Field Engineering, PLA University of Science and Technology, Nanjing, China \\ ${ }^{*}$ Corresponding author
}

\begin{abstract}
Camouflage as one of the effective way that against modern detection and precision strike and improving the survival ability of military targets is important content of combat. The digital camouflage can break the boundary of a camouflaged target between different colors, and make it blurred and broken, So as to attain a better camouflage effect. This paper proposes a new algorithm of camouflage design. It would first extract the main color after the transformation of color space, then use the Mathematical Morphology to delete the small spots in the picture, finally create digital camouflage with the color clustering way to replace the similar color with the selected main color. The experimental result shows that, the camouflage is easier to blend with the natural background than the traditional camouflage, and achieves a good effect of the optical camouflage.
\end{abstract}

Keywords-mathematical morphology; camouflage pattern; color space transformation; color clustering

\section{INTRODUCTION}

Camouflage is the use of paint, dye and other materials to change targets, screen and background color and pattern of camouflage [1, 2]. Camouflage can be divided into protective camouflage, deformation camouflage, imitation camouflage, camouflage of deformation is composed of several kinds of irregular shape blotches of multicolor camouflage, is mainly used for camouflage military vehicles and other activities. Deformation of the traditional camouflage mainly against the visible light and near infrared band, advanced deformation camouflage with function of thermal infrared camouflage, camouflage spots have different radiation characteristics of different colors, so that the target heat maps represent different spots, gray level, to distort the target contour, the purpose of reduce the striking feature [3].

Traditional camouflage pattern design mainly depends on the artificial experience, contrast background image and color board design camouflage design and selection of color. ${ }^{[2]}$ Its volume and high skills and experience to the design requirements, subjectivity is very strong. The development of image processing technology makes using computer-assisted camo design more and more common and attention. Based on the application of MATLAB software in this paper, combined with color clustering and morphological processing method, realized the extraction of camouflage color and quickly generate camouflage pattern. As far as possible to reduce unnecessary errors, in front of the clustering, adopt the most conforms to the human eye visual perception of HSV color space, morphology and applied, such as erode, opening operation and closing operation method further processing, end up with more practical camouflage pattern. The simulation results prove the effectiveness of the algorithm.

In this paper, on the basis of research of various kinds of camouflage pattern generation method, is proposed based on mathematical morphology method and the main color clustering and replace the camouflage pattern generation algorithm. The purpose of this research is to get a kind of can according to specific background camouflage effect ideal program aided by computer generated rapidly camouflage pattern.

Taking photos of target in the background by camera, using the MATLAB program, based on the HSV color space, the quantized color histogram, as far as possible to make it close to the human visual perception, to eliminate the digital color and communication barriers between traditional paint color, and then select any target area, color clustering, extract the main color, with the main color of color replacement, and then the main morphological processing color design, remove small spots, generate camouflage pattern, and finally the design cover to the corresponding position of the original image, edge detection and artificial visual inspection, analysis of their camouflage effect

\section{MATHEMATICAL MORPHOLOGY}

Mathematical morphology this name from shape research. This method also illustrates a fact that in many machine vision algorithm design, according to the shape to think the problem is the most natural, is also the most easily. Thinking approach helps morphology based on shape or graphics. Morphological methods in image information is the basic unit of binary pixels [4-7].

Any two binary image of A and B is A binary image, namely $A$ and $B$ are 1 set of image point $p[6]$ :

$$
A \cap B=\{p \mid p \in A, p \in B\}
$$

A and B and is A binary image, is A or B or both in A collection of all image points $\mathrm{p} 1$, with the symbol for:

$$
A \cup B=\{p \mid p \in A \quad \text { or } p \in B\}
$$


Set $\mathrm{B}$ is the value of binary image (all the pixels are 1 ), is a binary image. A complement is A 1 to 0 after the exchange of binary image, namely:

$$
\bar{A}=\{p \mid p \in \Omega, p \notin A\}
$$

Label for [I, j] and [k, l] two pixel points p and q, the vector and is labeled [I and k, j and l] pixel p and q. Vector is labeled [I - k, j - l] the pixels of $\mathrm{p}-\mathrm{q}$.

If $\mathrm{A}$ is a binary image, $\mathrm{p}$ is one of the binary image pixels, then a binary image by being $\mathrm{p}$ after translation type said:

$$
A_{p}=\{a+p \mid a \in A\}
$$

The binary image A is a pixel translation refers to the origin to a point $\mathrm{p}$ to $\mathrm{p}$.

Known binary image, if it is binary image pixel value is 1 point in translation, is A by B translation and called A is B dilate, namely.

$$
A \oplus B=\bigcup_{b_{i}} A_{b_{i}}
$$

Dilate has the associative, commutative. So, in dilate of the sequence of steps, finished the operation order is not important. This will allow us to a complex shape into a few simple shapes, and then reassembled as dilate sequence

Erode is the opposite process of dilate. Binary image after binary image $\mathrm{B}$ erode in a point $\mathrm{p}$ is still one of the necessary and sufficient condition is: after the translation top, B 1 pixel is also in A 1 pixel. A is B erode under the available type said:

$$
A \Theta B=\left\{p \mid B_{p} \subseteq A\right\}
$$

Binary image Images are often rules, is applied to the image of a probe, also known as structural units. Erode plays a very important role in many applications. Structural units of an image erode will generate a containing all the location of the structure of the yuan.

Dilate and erode shows the dual characteristics of geometric rather than logic, this feature also includes geometric complementarity and logical complementary. Binary image geometry complementary called its reflection. Binary image is the reflection of B and B symmetrical about the origin of binary image, namely:

$$
B^{\prime}=\{-p \mid p \in B\}
$$

Dilate and the duality of erode by the following relation said:

$$
\overline{A \oplus B}=\bar{A} \Theta B^{\prime}
$$

$$
\overline{A \Theta B}=\bar{A} \oplus B^{\prime}
$$

Basic operation of mathematical morphology can be combined into a very complex operation. Yuan (probe) with the same structure after erode dilate can remove all areas smaller than structural units of pixels, and leave the rest, this sequence is called "open" operation. Such as, with a circular probe will delete all smaller than the probe area, suppress additive airspace details of the filter. Contrary to the processing sequence is the process of dilate after erode, known as the "closed" operations, this order will fill the holes smaller than probe and concave shape area.

\section{COLOR CLUSTERING}

RGB color space and human eye perception differences, such as the distance of 50 two color $(0,0,0)$ and $(50,0,0)$ by the naked eye identification for the same black. ${ }^{[1]}$ And distance of 50 two colors $(200,50,0)$ and $(200,100,0)$ is the difference between a lot of red and yellow. HSV color space and human visual characteristic is similar, more can highlight color difference on the vision. HSV color space is divided into: tonal $\mathrm{H}$, saturation $\mathrm{S}$, brightness $\mathrm{V}$ three component, in this subject has its unique advantages in image processing application.

According to the statistical analysis of HSV color space image color interval, when the brightness $\mathrm{V}<0.15 \mathrm{~s}$, believed to be black, quantitative for $\mathrm{H}=0, \mathrm{~V}=0, \mathrm{~s}=0$, When $\mathrm{V}>0.85$, saturation $\mathrm{S}<0.1$, is considered to be white, quantitative for $\mathrm{H}$ $=0, \mathrm{~S}=1, \mathrm{~V}=1$, other area for color area. Based on the characteristics of visual psychology, the three components of the HSV color space in accordance with the 12, 5, 5 area quantified, after quantification, the HSV color space is divided into 300 color range, expressed in the middle of the corresponding color to each interval [1].

Results according to the quantitative, the three color component of $\mathrm{H}, \mathrm{S}, \mathrm{V}$ a characteristic vector $\mathrm{Q}$ for synthesis [1].

$$
\mathrm{Q}=25 \mathrm{H}+5 \mathrm{~S}+5 \mathrm{~V}
$$

$\mathrm{Q}=0,1, \ldots 299$, corresponding to each color range, $\mathrm{Q}$ is the only, as shown in the figure below, tonal $\mathrm{H}$ to quantify for five main areas of color, respectively is a red area $(\mathrm{Q}=0-24)$. The three yellow area $(\mathrm{Q}=255-99)$, four green area $(\mathrm{Q}=100-199)$, three blue area $(\mathrm{Q}=200-274)$, and a purple area $(\mathrm{Q}=275-299)$

According to the principle of the above extraction color in the picture, through the way of the shortest distance clustering, calculate the percentage of each color, choose five kinds of color as the main color.

In this paper, based on the theoretical basis is color clustering. Color clustering method is quantitative in HSV color space for 300 interval of the histogram, ordinate to color the number of pixels.

Abscissa to color range of partition. As shown in Figure 1: 

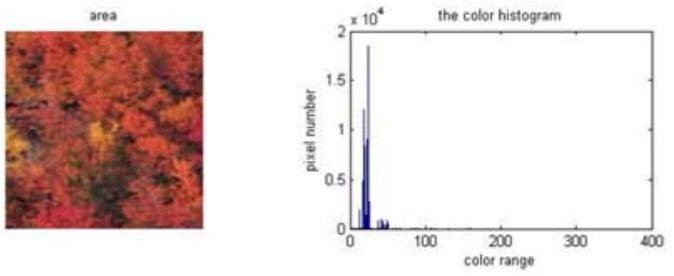

FIGURE I. COLOR CLUSTERING

The choice of color clustering, quantified the pixel number of the color of the top five, as a camouflage pattern design of the five main color.
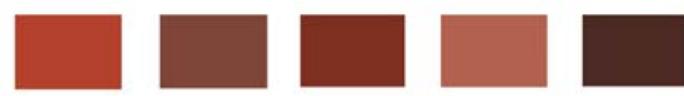

\section{$(R, G$, Bi $\quad[180,65,44) \quad(127,69,57) \quad(126,47,32) \quad(178,97,80 ; \quad[76,41,35)$}

FIGURE II. MAIN COLOR EXTRACTION

Used to extract the five main color, color of target area image substitution, the principle of replacement as the difference between the original image pixel color with the main color, with the smallest difference primary color to replace, end up with only five kinds of main color the main color design, which is ss shown in Figure 3.

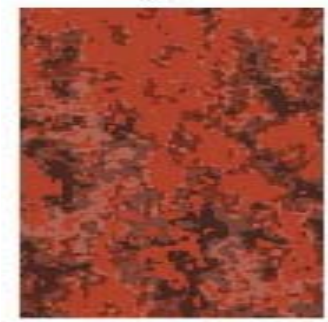

FIGURE III. MAIN COLOR REPLACEMENT

\section{CAMOUFLAge PATTERn DESIGN}

Using mathematical morphology method to the color design for the first time processing, get the preliminary processing design, in order to obtain more effective camouflage effect, on the basis of initial processing images, morphological processing again, secondary processing results are obtained. According to the results of the experiment many times, if again three processing, effect is not too big change, therefore, this paper only discuss the results of the analysis of the secondary processing figure. The result is shown in Figure 4.
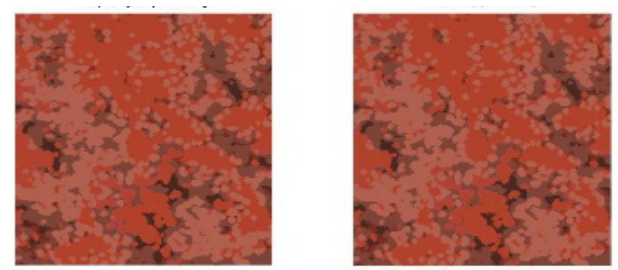

FIGURE IV. MORPHOLOGICAL PROCESSING

The morphological processing, primary processing of the openness of the dilate, erode, computing, four methods of closed operation, secondary processing is based on the processing for the first time, again, in turn, dilate, erode, opening operation and closing operation, eventually get total of 16 secondary processing result. Here just choose one way that first dilate processing, secondary processing as an example.

\section{The Simulation Results AND ANALysis}

The simulation effect chart is as shown in Figure 5.

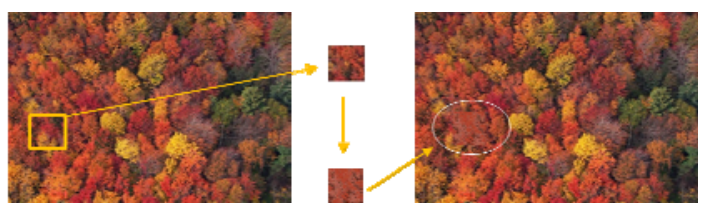

(a) background image (b) generate design (c) simulation results FIGURE V. SIMULATION RESULT

Observe Figure 5 can be found that using the algorithm to generate camouflage pattern, its color and background color close, design also similar and the distribution of the background, effectively reduces the significance of the target. To better achieve the goal of integration of target and background. And the algorithm can get the exact color and pattern shape, avoid the past rely on artificial experience in design because of the subjective factors of the main color selection is not allowed or camouflage pattern with the background. In order to improve the camouflage pattern and background better similarity, in this paper, the algorithm can be designed according to the practical background of the situation, change the color of the species, it is realized to achieve better camouflage effect in different special occasions. The simulation design of camouflage effect, using two methods in this paper. One is a MATLAB program of the edge detection algorithm, the simulation effect of alignment design and background, another is to use artificial visual way, this experiment is 20 people rated the simulation effect of different processing design, the final statistical data, then compare the optimal morphology processing method, which is shown in Figure 6.

Sobel edge detection algorithm in extracting the edge of the algorithm is effect is good method, in this article chooses the algorithm for the background image edges and simulation rendering [8-11].

After comparing different treatment effect of edge detection results, with a background image fusion effect best is the first processing method: erode and open computing. The rest of the three methods can be seen at the edge of the target area obviously has a square shape. In this experiment, and then select the personnel of 20 normal vision, visual observation on Figure 6. Five pattern shown in Figure 6, followed by erode and open operation pattern and closing operations and open operations, dilate and open operation pattern, open operations and open operations, military desert camouflage pattern. The researchers observed Camouflage effect of five kinds of patterns in the background, which is shown in Figure 7. 


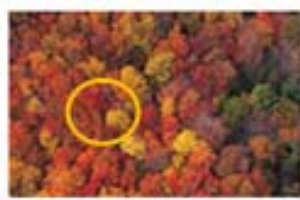

(k) Erode and opes operation

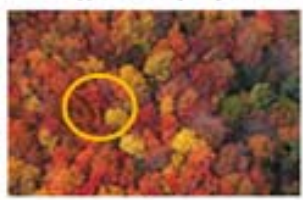

(E) Oole and open operatich

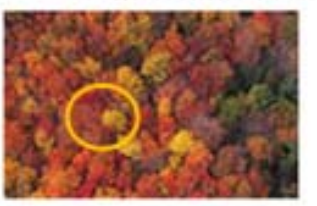

igi Dlate and epen coprations

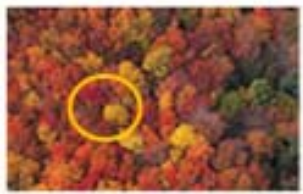

11) Twice epen sperations

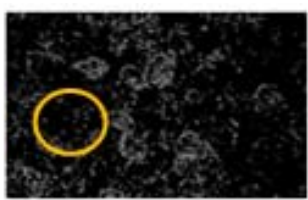

(6) foge Getection

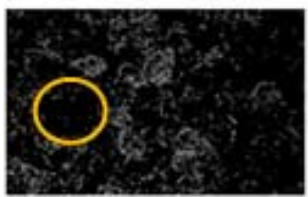

n) Edse detection

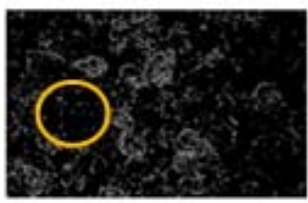

(i) Edgedetection

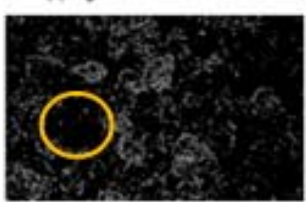

wiv Edge detection
FIGURE VI. EDGE DETECTION

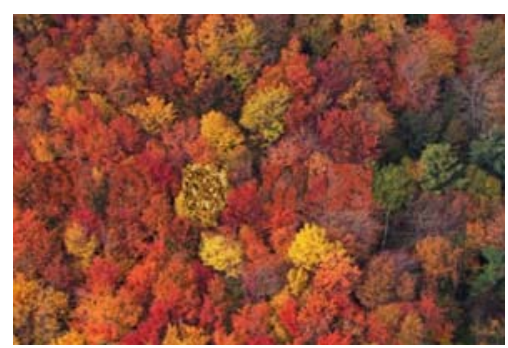

FIGURE VII. VISUAL TEST PATTERN

20 experimental personnel by subjective feeling about their scores think camouflage effect good camouflage pattern. Number is as follows in Table 1.

TABLE I. CAMOUFLAGE EFFECT SCORES

\begin{tabular}{|c|c|c|c|c|c|}
\hline leve & $\begin{array}{c}\text { hard } \\
\text { to tell }\end{array}$ & $\begin{array}{c}\text { Not } \\
\text { easy to } \\
\text { tell }\end{array}$ & $\begin{array}{c}\text { Easy } \\
\text { to } \\
\text { tell }\end{array}$ & $\begin{array}{c}\text { not } \\
\text { difficult to } \\
\text { tell }\end{array}$ & $\begin{array}{c}\text { Easy to } \\
\text { distinguish }\end{array}$ \\
\hline $\begin{array}{c}\text { Erode and } \\
\text { open } \\
\text { operation }\end{array}$ & 15 & 5 & 0 & 0 & 0 \\
\hline $\begin{array}{c}\text { Close and } \\
\text { open } \\
\text { operations }\end{array}$ & 7 & 8 & 4 & 1 & 0 \\
\hline $\begin{array}{c}\text { dilate and } \\
\text { open } \\
\text { operations }\end{array}$ & 4 & 7 & 8 & 1 & 0 \\
\hline $\begin{array}{c}\text { twice open } \\
\text { operations }\end{array}$ & 8 & 4 & 6 & 2 & 0 \\
\hline $\begin{array}{c}\text { Military } \\
\text { desert } \\
\text { camouflage }\end{array}$ & 0 & 2 & 3 & 13 & 2 \\
\hline
\end{tabular}

According to statistics, can directly come to the conclusion that the visual condition of the same circumstances, camouflage pattern effect after erode and open computing optimal, in optical observation under the camouflage effect is best.

\section{CONCLUSION}

In order to get accurate and stable effect of camouflage color design, reduce artificial drawing of error and tedious work, puts forward the quantitative color histogram based on HSV color space, and color clustering method is used to extract the main color of target area. To make the camouflage pattern more neat practical, using mathematical morphology to the main color design further to deal with the small spots. Simulation experiments as a first step, is to use different methods of mathematical morphology, the main color design process, verify the morphology of pattern shape. Simulation results in the second step, through the study of the simulation results of different kinds of camouflage pattern analysis, verify the algorithm design of camouflage pattern effect in this paper.

Comprehensive comparison of two kinds of simulation design of camouflage effect analysis method, use erode and open operation, the effect is the best. The qualitative analysis and artificial visual conditions are ideal camouflage effect. This paper by using the algorithm is feasible and can be quickly and effectively generate camouflage pattern with high background alignment. From the simulation results of the experiment can draw the following conclusion: Using mathematical morphology, to design the shape of the processing effect is best, shape, minimal disruption. USES color clustering and the main color replacement and mathematical morphology method can accurately extract the Main color of target area, with high degrees of similar background generation and camouflage pattern. Different methods of mathematical morphology, design is affected by the effect of processing, erode and open computing pattern after the most ideal effect.

\section{REFERENCES}

[1] Y. H. Huang, W. Y. Wu, Y. C. Gong and H. Y. Du, "Improvement and Application of Color Histogram," Electro-optic Techonlogy Application, vol.26, pp.76-80, 2011.

[2] Z. Y. Liu, Z. R. Wang, J. C. Wang and X. Q. Sun, "Design method of imitated pattern painting based on main color clustering,” , Laser \& Infrared, vol. 39, pp. 793-796, 2009.

[3] D. Mark, W. Paul and L. Clayton, "Defeating Camouflage and Finding Explosives Through Spectral Matched-Filtering of Hyperspectral Imagery,” SPIE, vol, 2933, pp. 109-118.

[4] .S. R. Sternberg, “Grayscale morphology”, Computer Vision, Graphics, and image processing, vol.35, pp. 333-335, 1986.

[5] J. Edmond, J. Ronald, T. Hugues T, "Mathematical morphology, a useful set of tools for image analysis," Statistics and Computing, vol.10, pp. 105-120, 2000.

[6] H. X. Yan, "Study on Algoithms of Image Edge Detection and Enhancement Based on Mathematical Morphology, ”JiLin University, 2009.

[7] E. Navon, O. Miller and A. Averbuch, "Color image segmentation based on adaptive local thresholds" Image and Vision Computing, vol.23, 2005.

[8] M. Herbin, N. Bonnet and P. Vautrot, "Aclustering method based on the estimation of the probability density function and on the skeleton by 
influence zones,” Pattern Recognition Letters, vol.17, pp. 1141-1150, 1996.

[9] B. Chanda, J. M. .K Kurrt, and Y. V. Panaja, "A multi-scale morphological edge deteclor,” Pattern Recognition, vol. 31, pp. 1469$1478,1998$.

[10] S. Giannarou and T. Stathaki, "Edge detection using quantitative combination of multiple operators," International Journal of Electronics and Communications, vol.61, pp. 546-550, 2007.

[11] S James and J. Lee, "Morphological Edge Detection," IEEE Journal of Robotics and Automation, vol.3, pp. 142-156, 1987. 